HU-EP-03/06

CERN-TH/2003-032

hep-th/0303016

\title{
Moduli Stabilization in Chiral Type IIB Orientifold Models with Fluxes
}

\author{
Ralph Blumenhagen ${ }^{1}$, Dieter Lüst ${ }^{1}$, Tomasz R. Taylor ${ }^{2, \star}$ \\ ${ }^{1}$ Humboldt-Universität zu Berlin, Institut für Physik, \\ Invalidenstrasse 110, 10115 Berlin, Germany \\ e-mail: blumenha, luest@physik.hu-berlin.de \\ ${ }^{2}$ CERN Theory Division, CH-1211 Geneva 23, Switzerland \\ e-mail: taylor@neu.edu
}

\begin{abstract}
We consider Type IIB orientifold models on Calabi-Yau spaces with three-form G-flux turned on. These fluxes freeze some of the complex structure moduli and the complex dilaton via an F-term scalar potential. By introducing pairs of D9- $\overline{\mathrm{D} 9}$ branes with abelian magnetic fluxes it is possible to freeze also some of the Kähler moduli via a D-term potential. Moreover, such magnetic fluxes in general lead to chiral fermions, which make them interesting for string model-building. These issues are demonstrated in a simple toy model based on a $\mathbb{Z}_{2} \times \mathbb{Z}_{2}^{\prime}$ orbifold.
\end{abstract}

${ }^{\star}$ On sabbatical leave from Department of Physics, Northeastern University, Boston, MA 02115, USA. 


\section{Introduction}

There exist many obstacles that string theory has to overcome in order to make contact with low-energy physics. The list of problems contains in particular the question of how to remove the huge vacuum degeneracy, i.e. how to fix the moduli that are typically present in any string compactification. Second, the light string modes must reproduce the spectrum of the standard model; hence realistic string vacua must naturally lead to chiral fermions. A third, very important issue is the problem of space-time supersymmetry breaking, which eventually has to be achieved without creating any new vacuum instabilities. With the advent of D-branes and also with the introduction of background fluxes in the internal space, some new perspectives in addressing these questions arose during the last few years.

First, in the context of intersecting brane world models [1-26] with type IIA D6-branes wrapped around 3-cycles of the internal Calabi-Yau space, it is possible to construct in a systematic way orientifold compactifications with standard model-like spectra with chiral fermions. Part of the space-time supersymmetry is preserved if the intersecting D6-branes wrap supersymmetric (special lagrangian) 3-cycles, which must be calibrated with respect to the same holomorphic 3-form as the O6-planes are. In general, the tension of the D6-branes and of the O6-planes introduces a vacuum energy, which is described by a Fayet-Iliopolous term in the language of $N=1$ supersymmetric field theory [16]. These D-terms depend only on (part of) the complex structure moduli, which can be fixed upon minimization of the potential. In the (T-dual) Type IIB mirror picture, one is dealing with magnetic gauge fluxes on the world-volume of D9-branes [2,20]. Since the Type IIB F-flux is integrated over 2-cycles of the Calabi-Yau space, the D-term potential now stabilizes (part of) the Type IIB Kähler moduli.

Another recent approach to moduli stabilization involves Type IIB background 3-form fluxes on the internal Calabi-Yau manifold, $G=\tau H_{3}+F_{3}$, where $H_{3}$ originates from the NS-NS sector, $F_{3}$ from the R-R sector and $\tau$ is the dilaton that "complexifies" the flux [27]. These so-called G-fluxes give rise to a scalar potential, which freezes many of the complex structure moduli of the Calabi-Yau and the dilaton. In addition, supersymmetry may get partially or completely broken. A very convenient way of analyzing the consequences of turning on G-fluxes is to use an effective superpotential that can be computed from the tendimensional kinetic terms for the 3-forms [28]. Using the effective F-term (super)potential, the vacuum structure of this type of flux compactifications was recently discussed in several papers [2944. Upon inspection of the induced potential, one realizes that turning on 
these fluxes on a compact Calabi-Yau space in a local way, i.e. such that $\int H_{3} \wedge F_{3}=0$, the minima of the potential are generically at those points in the Calabi-Yau moduli space where the geometry degenerates and supersymmetry is restored [28]. So far, partial supersymmetry breaking was only shown to be possible at certain (conifold) points of a non-compact Calabi-Yau space. As we will discuss in this paper, by choosing non-local fluxes with $\int H_{3} \wedge F_{3} \neq 0$, partial supersymmetry breaking and moduli stabilization can also be achieved at points where the compact Calabi-Yau space is non-degenerate.

The main aim of this paper is to combine the G-flux compactifications with the scenario of Type IIA intersecting branes or, respectively, with Type IIB magnetic gauge fluxes on D9-branes. For concreteness, we will apply the general formalism to a Type IIB $\mathbb{Z}_{2} \otimes \mathbb{Z}_{2}^{\prime}$ orientifold model with O3- and three sets of O7-planes. We turn on both non-trivial G-flux through 3-cycles of the orbifold space and non-trivial abelian magnetic F-fluxes through 2-cycles supported on pairs of D9- $\overline{\mathrm{D} 9}$ branes. Thus, we cancel the localized tadpoles of both the O3- and the O7-planes in a non-local way. At leading order, we will show that the F-flux through 2-cycles can cancel all R-R tadpoles, with chiral fermions and part of the Kähler moduli frozen via a D-term potential. In addition, the G-fluxes through 3-cycles can be chosen in such a way that additional complex structure moduli are stabilized by an F-term potential. Since we are choosing the G-fluxes in a non-local way, $\int H_{3} \wedge F_{3} \neq 0$, the Chern-Simons term in the ten-dimensional effective IIB action will provide a G-flux contribution to the Ramond charges, which has to be canceled by the various non-local D-brane charges together with the negative $\mathrm{R}$ - $\mathrm{R}$ charge of the orientifold planes.

Let us emphasize that in this paper we are only working in leading order in string and sigma model perturbation theory. In particular, since we are not cancelling the D7brane charge locally, we neglect the significant back-reaction on the dilaton and on the background geometry, which, as we learned from F-theory, is expected to lead to nonRicci-flat manifolds.

The paper is organized as follows. In the next section we briefly define our Type IIB $\mathbb{Z}_{2} \otimes \mathbb{Z}_{2}^{\prime}$ orientifold model. Then, in section 3, we introduce the 3-form G-fluxes, compute their R-R tadpoles, and discuss some general features of G-flux-induced potentials. We stress the importance of mutual "non-locality" of the R-R and NS-NS fluxes in avoiding ground states corresponding to degenerate Calabi-Yau manifolds. In the next section, we work out some details of the $\mathbb{Z}_{2} \otimes \mathbb{Z}_{2}^{\prime}$ orientifold compactification and discuss the pattern of complex structure moduli stabilization and the issue of supersymmetry breaking. Finally, in section 5, we introduce D9- $\overline{\mathrm{D} 9}$ branes with F-fluxes and present brane configuration 
with chiral fermions, which satisfy all tadpole conditions. It should be mentioned that these models are not realistic; however it neatly demonstrates that models with G-fluxes and D9- $\overline{\mathrm{D} 9}$ branes with F-fluxes (or intersecting D6-branes in the T-dual picture) can be constructed in such a way that the chiral fermions survive and both types of moduli can be at least partially stabilized. We hope that our construction can serve as a template for more realistic model-building.

\section{The Type IIB orientifold model}

In [35] it was explicitly shown how turning on appropriate 3 -form fluxes in a toroidal orientifold $\left[\Omega R(-1)^{F_{L}}\right]$ model can lead to supersymmetry breaking while freezing some of the complex structure moduli, Kähler moduli, and the dilaton. At the level of the fourdimensional effective action, the freezing of the complex structure moduli and the dilaton was due to the F-term potential [28]. After turning on this G-flux, the Chern-Simons term in the ten-dimensional Type IIB effective action produces a tadpole for the R-R 4-form potential. In general, additional D3-branes had to be present in order to satisfy the R-R tadpole cancellation condition for the 4-form. The resulting gauge theory on the D3-branes in the example discussed in [35] is always non-chiral. In view of applications to realistic string model building, it is desirable to generalize such flux compactifications to cases admitting chiral gauge theories.

It is known that a large class of chiral models is given by intersecting brane world models or, in their T-dual version, by D-branes with magnetic fluxes [45, 46]. Since we would like to have the possibility to preserve supersymmetry, we are led to flux compactifications on orientifolds of Calabi-Yau threefolds. To be more precise, in this paper we consider a simple orientifold model, namely we choose the Calabi-Yau to be given by the orbifold

$$
X=\frac{T^{6}}{\mathbb{Z}_{2} \times \mathbb{Z}_{2}^{\prime}} .
$$

The two $\mathbb{Z}_{2}$ operations are given by

$$
\theta:\left\{\begin{array}{l}
z^{1} \rightarrow-z^{1} \\
z^{2} \rightarrow-z^{2} \\
z^{3} \rightarrow z^{3}
\end{array} \quad, \quad \theta^{\prime}:\left\{\begin{array}{l}
z^{1} \rightarrow z^{1} \\
z^{2} \rightarrow-z^{2} \\
z^{3} \rightarrow-z^{3}
\end{array}\right.\right.
$$

on the three complex coordinates of $T^{6}=T^{2} \times T^{2} \times T^{2}$. These data do not specify the orbifold completely, as we have the freedom of introducing a discrete torsion, $\epsilon= \pm 1$ 
in the $\mathbb{Z}_{2}$ twisted sectors. Following [47] we associate the model without discrete torsion, $\epsilon=+1$, to Hodge numbers $\left(h_{21}, h_{11}\right)=(51,3)$ and the model with discrete torsion, $\epsilon=-1$, to the mirror with $\left(h_{21}, h_{11}\right)=(3,51)$. For $\epsilon=+1$, in the three $\mathbb{Z}_{2}$ twisted sectors, the 3 -cycles survive the projection, while for $\epsilon=-1$, the 2-cycles survive. In the case with discrete torsion, all 3-cycles on $X$ derive from 3-cycles on the ambient $T^{6}$. The massless bosonic modes in the untwisted sector arise as follows. From the ten-dimensional graviton one gets the four-dimensional graviton, $g_{\mu \nu}$, and 9 scalars from the internal components of the metric $g_{a b}$. Six of these scalars form 3 chiral multiplets containing the 3 complex structure moduli $\mathcal{T}^{i}$. The 3 remaining scalars combine with 3 additional scalars from the internal components of the R-R 4-form, $\left(C_{4}\right)_{a b c d}$, into 3 chiral multiplets, $C^{I}+i \mathcal{K}_{s}^{I}$ containing the untwisted Kähler moduli $\mathcal{K}_{s}^{I}$. Finally, one also gets the complex dilaton multiplet $C_{0}+i e^{-\phi}$. This untwisted massless spectrum is enhanced by 48 chiral multiplets containing the Kähler moduli related to the fixed points of the $\mathbb{Z}_{2}$ actions. In the case without discrete torsion, the chiral multiplets from the twisted sectors are related to 48 additional complex structure moduli.

In order to introduce objects that contribute negatively to the $\mathrm{R}-\mathrm{R}$ tadpole, we now take the additional quotient by $\Omega R(-1)^{F_{L}}$, where $R$ reflects all three complex coordinates: $z^{I} \rightarrow-z^{I}$. This breaks supersymmetry in the closed string sector down to $N=1$ and introduces 64 O3-planes located at the fixed points of $R$ in addition to three sets of 4 O7-planes located at the fixed locus of $R \theta, R \theta^{\prime}$ and $R \theta \theta^{\prime}$.

There is a subtlety at this point, which deserves some clarifying comments. As shown in [47], the perturbative orientifold with discrete torsion is forced to contain exotic orientifold planes in order to satisfy the cross-cap constraint 1

$$
\int d l\left\langle\Omega R(-1)^{F_{L}} \theta\left|e^{-l \mathcal{H}_{c l}}\right| \Omega R(-1)^{F_{L}} \theta^{\prime}\right\rangle=\int \frac{d t}{t} \operatorname{Tr}_{\theta \theta^{\prime}}\left(\Omega R(-1)^{F_{L}} \theta e^{-2 \pi t H}\right) .
$$

Such exotic orientifold planes have positive charge and tension. More concretely, an odd number of the four classes of orientifold planes have to be of type $\mathrm{Op}^{(+,+)}$. Note that the intersecting brane world model discussed in [13,14 is T-dual to the Type IIB orientifold model without discrete torsion.

To proceed farther, we now allow turning on non-trivial 3-form fluxes, which will contribute to the 4 -form tadpole. In order to cancel the three 8-form tadpoles, we have to introduce additional D7-branes. Since we would like to discuss chirality, we allow more generally the presence of D9-branes with magnetic fluxes.

1 We thank G. Pradisi for a discussion on this point. We are indepted to A. Uranga for pointing out an error in an earlier version of the paper. 


\section{Three-form fluxes}

In this section, we discuss the effect of turning on NS-NS and R-R 3-form fluxes on the internal Calabi-Yau manifold. We are using the conventions and notation of [28] and 48 .

\section{1. $R$ - $R$ tadpole}

The Chern-Simons term in the Einstein-frame effective Type IIB action looks like

$$
S_{C S}=\frac{1}{2 \kappa_{10}^{2}} \int_{M_{4} \times X} \frac{C_{4} \wedge G \wedge \bar{G}}{4 i \operatorname{Im}(\tau)} .
$$

Recall that $G=\tau H_{3}+F_{3}$, where $H_{3}$ comes from the NS-NS sector, $F_{3}$ from the R-R sector and $\tau=C_{0}+i e^{-\phi}$ is the dilaton which "complexifies" the flux. The ten-dimensional gravitational coupling is given in terms of $\alpha^{\prime}$ as $\kappa_{10}^{2}=\frac{1}{2}(2 \pi)^{7}\left(\alpha^{\prime}\right)^{4}$. From (3.1) it is clear that turning on a non-trivial G-flux leads to a tadpole for the 4 -form $C_{4}$. In fact, the contribution to the tadpole is given by

$$
N_{\text {flux }}=\frac{1}{2 \kappa_{10}^{2} \mu_{3}} \int_{X} H_{3} \wedge F_{3},
$$

with $\mu_{p}=(2 \pi)^{-p}\left(\alpha^{\prime}\right)^{-(p+1) / 2}$. To describe the fluxes we assume that we have an integral basis of the homology $H_{3}(X, \mathbb{Z})$ with the intersections $A^{\Lambda} \cap A^{\Sigma}=B_{\Lambda} \cap B_{\Sigma}=0$ and $A^{\Lambda} \cap B_{\Sigma}=\delta_{\Sigma}^{\Lambda}\left(\Lambda, \Sigma=0, \ldots, h_{21}\right)$. In terms of the Poincaré dual basis of $H^{3}(X, \mathbb{Z})$, $\left(\alpha_{\Lambda}, \beta^{\Lambda}\right)$, the covariantly constant $(3,0)$ form can be expanded as

$$
\Omega_{3}=X^{\Lambda} \alpha_{\Lambda}-F_{\Lambda} \beta^{\Lambda}
$$

with

$$
X^{\Lambda}=\int_{A^{\Lambda}} \Omega_{3}, \quad F_{\Lambda}=\int_{B_{\Lambda}} \Omega_{3},
$$

where the periods $X^{\Lambda}$ and $F_{\Lambda}$ are functions of the complex structure moduli $\mathcal{T}^{i}(i=$ $\left.1, \ldots, h_{21}\right)$. Due to the Bianchi identities, the three-forms $H_{3}$ and $F_{3}$ are closed, therefore in cohomology they can be expressed as integer linear combinations

$$
\begin{aligned}
& \frac{1}{(2 \pi)^{2} \alpha^{\prime}} H_{3}=e_{\Lambda}^{1} \beta^{\Lambda}+m^{1 \Lambda} \alpha_{\Lambda} \\
& \frac{1}{(2 \pi)^{2} \alpha^{\prime}} F_{3}=e_{\Lambda}^{2} \beta^{\Lambda}+m^{2 \Lambda} \alpha_{\Lambda} .
\end{aligned}
$$

Thus the complex three-form flux can be written as

$$
\frac{1}{(2 \pi)^{2} \alpha^{\prime}} G=e_{\Lambda} \beta^{\Lambda}+m^{\Lambda} \alpha_{\Lambda}
$$

with $e_{\Lambda}=\tau e_{\Lambda}^{1}+e_{\Lambda}^{2}$ and $m^{\Lambda}=\tau m^{1 \Lambda}+m^{2 \Lambda}$. In this notation, the tadpole (3.2) becomes

$$
N_{\text {flux }}=m \times e,
$$

with $m \times e=m^{1 \Lambda} e_{\Lambda}^{2}-m^{2 \Lambda} e_{\Lambda}^{1}$. 


\subsection{The scalar potential}

The kinetic term for the G-flux

$$
S_{G}=-\frac{1}{4 \kappa_{10}^{2} \operatorname{Im}(\tau)} \int_{X} G \wedge \star_{6} G,
$$

when integrated over the internal manifold, gives rise to a scalar potential, which has been computed in [28]. Note, that we use the convention that the Hodge star operator involves also complex conjugation. Working in the $\left(\alpha_{\Lambda}, \beta^{\Lambda}\right)$ basis the computation is straightforward when one uses the following action of the Hodge star operator

$$
\begin{aligned}
& \star \alpha=A \alpha+B \beta \\
& \star \beta=C \alpha+D \beta,
\end{aligned}
$$

where the four matrices $A, B, C, D$ can be expressed in terms of the period matrix $\mathcal{N}$

$$
\begin{aligned}
& A=-D^{T}=(\operatorname{Re} \mathcal{N})(\operatorname{Im} \mathcal{N})^{-1} \\
& B=-\operatorname{Im} \mathcal{N}-(\operatorname{Re} \mathcal{N})(\operatorname{Im} \mathcal{N})^{-1}(\operatorname{Re} \mathcal{N}) \\
& C=(\operatorname{Im} \mathcal{N})^{-1}
\end{aligned}
$$

In a symplectic basis in which the prepotential $F$ exists 48,

$$
\mathcal{N}_{\Lambda \Sigma}=\bar{F}_{\Lambda \Sigma}+2 i \frac{\operatorname{Im}\left(F_{\Lambda \Gamma}\right) \operatorname{Im}\left(F_{\Sigma \Delta}\right) X^{\Gamma} X^{\Delta}}{\operatorname{Im}\left(F_{\Gamma \Delta}\right) X^{\Gamma} X^{\Delta}}
$$

where $F_{\Lambda \Sigma}=\partial^{2} F / \partial X_{\Lambda} \partial X_{\Sigma}$.

The scalar potential resulting from (3.8) can be expressed as

$$
V=-\frac{\mu_{3}}{2 \operatorname{Im} \tau}\left[m(\operatorname{Im} \mathcal{N}) \bar{m}+(e+m \operatorname{Re} \mathcal{N})(\operatorname{Im} \mathcal{N})^{-1}(\bar{e}+\bar{m} \operatorname{Re} \mathcal{N})\right]
$$

Since the period matrix depends on complex structure moduli, $V$ is a function of $\mathcal{T}^{i}$ and $\tau$. This scalar potential can also be rewritten as

$$
V=-\frac{\mu_{3}}{2 \operatorname{Im} \tau}\left[(e+m \overline{\mathcal{N}})(\operatorname{Im} \mathcal{N})^{-1}(\bar{e}+\bar{m} \mathcal{N})\right]+\mu_{3} m \times e,
$$

or as

$$
V=-\frac{\mu_{3}}{2 \operatorname{Im} \tau}\left[(e+m \mathcal{N})(\operatorname{Im} \mathcal{N})^{-1}(\bar{e}+\bar{m} \overline{\mathcal{N}})\right]-\mu_{3} m \times e .
$$

Recall that in our conventions, one requires $\operatorname{Im} \mathcal{N}<0$ in the physical domain of positivedefinite kinetic energy terms, while $\operatorname{Im} \tau>0$. 
In [28], only the "local" case of $N_{f l u x}=m \times e=0$ was considered. Then it is easy to see that no stable minima of the potential exist, except at some singular points (or limits) where the period matrix degenerates. In order to understand the origin of this result and the "non-local" case of $m \times e \neq 0$, we first notice that there are two obvious candidates for the minima, at

$$
e_{\Lambda}+m^{\Sigma} \overline{\mathcal{N}}_{\Sigma \Lambda}=0
$$

and at

$$
e_{\Lambda}+m^{\Sigma} \mathcal{N}_{\Sigma \Lambda}=0
$$

After multiplying (3.15) by $\bar{m}^{\Lambda}$ and taking the imaginary part, we obtain

$$
m(\operatorname{Im} \mathcal{N}) \bar{m}=-\operatorname{Im} \tau(m \times e)
$$

hence Eq. (3.15) can be satisfied in the physical positivity domain only if $m \times e>0$. By a similar argument, $m \times e<0$ is a necessary condition for the existence of a non-degenerate solution of Eq. (3.16). Hence for a given sign of $m \times e$, only one of the two equations (3.15) and (3.16) can be solved. Note that the potential is always positive at the minimum point: $V_{\text {min }}=\mu_{3}|m \times e|=\mu_{3}\left|N_{\text {flux }}\right|$.

In [28] is was shown that the first term in (3.13) can be understood as the F-term scalar potential arising from the superpotential [49,50]

$$
W=\frac{1}{\sqrt{2} \kappa_{10}} \int_{X} \Omega_{3} \wedge G=\sqrt{\mu_{3}}\left(e_{\Lambda} X^{\Lambda}+m^{\Lambda} F_{\Lambda}\right) .
$$

In fact, by using the identities 48 $F_{\Lambda}=\mathcal{N}_{\Lambda \Sigma} X^{\Sigma}, D_{i} F_{\Lambda}=\overline{\mathcal{N}}_{\Lambda \Sigma} D_{i} X^{\Sigma}$ and the tree-level Kähler potential, one can rewrite (3.13) as 目

$$
V=\mu_{3} e^{[K(z, \bar{z})+\tilde{K}(\tau, \bar{\tau})]}\left[G^{i \bar{j}} D_{i} W D_{\bar{j}} \bar{W}+G^{\tau \bar{\tau}} D_{\tau} W D_{\bar{\tau}} \bar{W}\right]+\mu_{3} m \times e .
$$

Assuming that (3.15) is satisfied,

$$
D_{i} W=D_{\tau} W=0
$$

the existence of a supersymmetric minimum is therefore not guaranteed, unless $W=0$ for some choice of fluxes. If the minimum is described by Eq. (3.16) instead of (3.15), it is convenient to replace (3.18) by an "almost holomorphic" superpotential $\widetilde{W}=W(e \rightarrow \bar{e}, m \rightarrow \bar{m})$,

${ }^{2}$ The $-3|W|^{2}$ term is canceled by Kähler derivatives of the CY volume hypermultiplet. 
now treating $\bar{\tau}$ as a chiral field. Such a superpotential generates the potential (3.14), up to the $m \times e$ constant term. It is easy to see that Eqs. (3.20) are satisfied for this new superpotential, with $\tau \rightarrow \bar{\tau}$.

We will see in section 5 that the topological $m \times e$ term in (3.19) plays an essential rôle in the computation of the Fayet-Iliopolous terms for the abelian gauge groups on the D-branes, which have to be introduced to cancel the $\mathrm{R}-\mathrm{R}$ tadpoles. The $m \times e$ term is proportional to the $\mathrm{R}-\mathrm{R}$ three-form charge, which already indicates that it is nothing else than the effective "D3-brane" tension of the G-flux.

Another, equivalent way of discussing supersymmetry breaking is by examining the supersymmetry transformations of fermions [35]. The condition for (at least one) unbroken supersymmetry can be succinctly summarized as the requirement that $G$ be a pure $(2, \overline{1})$ [or $(1, \overline{2})]$ form. Is this condition satisfied in a vacuum described by Eqs. (3.15) or (3.16)? It is easy to see that Eq. (3.15) is equivalent to the condition that $G$ be "imaginary antiself dual", i.e. $\star G=-i \bar{G}$; hence, in addition to the $(2, \overline{1})$ part, it may also contain $(0, \overline{3})$; the latter may vanish, though, for a particular choice of fluxes, which ensures $W=0$ at the minimum. Similarly, Eq. (3.16) describes an "imaginary self-dual" flux configuration, $\star G=i \bar{G}$, a $(1, \overline{2})$ form with a possible $(3, \overline{0})$ admixture that vanishes if $\widetilde{W}=0$. Note that the additional condition for supersymmetry, namely that the $G$ flux has to be primitive,

$$
J \wedge G=0
$$

can be satisfied on a Calabi-Yau manifold, as there are no cohomologically non-trivial closed 5-forms.

We conclude that a stable vacuum can only exist in the "non-local" case of $m \times e \neq 0$ i.e. with a non-vanishing $N_{\text {flux }}$. This typically leads to a complete supersymmetry breakdown; however, one (or more) supersymmetry may survive if the moduli satisfy one additional constraint. Note that the number of equations contained in (3.15) [as well as in (3.16)] is equal to the number of undetermined moduli (including the dilaton), and that moduli stabilization is therefore expected to occur for a generic pattern of fluxes while unbroken supersymmetry would take place only in some special cases. In the next section we will describe examples illustrating both kinds of situations.

\section{4. $\mathbb{Z}_{2} \otimes \mathbb{Z}_{2}^{\prime}$ Orbifolds}

As an example we now apply the general formalism presented in section 2 to our $\mathbb{Z}_{2} \otimes \mathbb{Z}_{2}^{\prime}$ orientifold. 


\subsection{Cohomological basis}

The following closed 3-forms on the toroidal ambient space are invariant under the $\mathbb{Z}_{2} \times \mathbb{Z}_{2}^{\prime}$ orbifold symmetry

$$
\begin{array}{ll}
\alpha_{0}=d x^{1} \wedge d x^{2} \wedge d x^{3} \quad & \beta^{0}=d y^{1} \wedge d y^{2} \wedge d y^{3} \\
\alpha_{1}=d y^{1} \wedge d x^{2} \wedge d x^{3} & \beta^{1}=-d x^{1} \wedge d y^{2} \wedge d y^{3} \\
\alpha_{2}=d x^{1} \wedge d y^{2} \wedge d x^{3} & \beta^{2}=-d y^{1} \wedge d x^{2} \wedge d y^{3} \\
\alpha_{3}=d x^{1} \wedge d x^{2} \wedge d y^{3} & \beta^{3}=-d y^{1} \wedge d y^{2} \wedge d x^{3} .
\end{array}
$$

These are Poincaré-dual to the obvious 3-cycles on $T^{6}$. Note that expanding $H_{3}$ and $F_{3}$ in terms of these eight 3 -forms guarantees that the 3 -form fluxes in (3.5) are invariant under the orientifold symmetry $\Omega R(-1)^{F_{L}}$.

There are three moduli, $\mathcal{T}^{i} \equiv \mathcal{R}^{i}+i \mathcal{I}^{i}, i=1,2,3$, which define the complex structure: $z^{i}=x^{i}+\mathcal{T}^{i} y^{i}$ (no summation over $i$ ) on the orbifold space. As usual, the holomorphic 3 -form

$$
\Omega_{3}=d z^{1} \wedge d z^{2} \wedge d z^{3}=X^{\Lambda} \alpha_{\Lambda}-F_{\Lambda} \beta^{\Lambda}
$$

defines the homogeneous coordinates $X^{\Lambda}$ and the derivatives $F_{\Lambda}=\partial_{\Lambda} F$ of the prepotential

$$
\begin{array}{ll}
X^{0}=1 & F_{0}=-\mathcal{T}^{1} \mathcal{T}^{2} T^{3} \\
X^{1}=\mathcal{T}^{1} & F_{1}=\mathcal{T}^{2} T^{3} \\
X^{2}=\mathcal{T}^{2} & F_{2}=\mathcal{T}^{1} T^{3} \\
X^{3}=\mathcal{T}^{3} & F_{3}=\mathcal{T}^{1} T^{2} .
\end{array}
$$

Therefore the prepotential is given by

$$
F=\frac{X^{1} X^{2} X^{3}}{X^{0}}=\mathcal{T}^{1} \mathcal{T}^{2} \mathcal{T}^{3}
$$

It is convenient to introduce the following basis of $(2, \overline{1})$ forms:

$$
\begin{aligned}
& a_{1}=d \bar{z}^{\overline{1}} \wedge d z^{2} \wedge d z^{3}, \\
& a_{2}=d z^{1} \wedge d \bar{z}^{\overline{2}} \wedge d z^{3}, \\
& a_{3}=d z^{1} \wedge d z^{2} \wedge d \bar{z}^{\overline{3}} .
\end{aligned}
$$


By using the prepotential (4.4) and Eq. (3.11), we obtain the following (symmetric) period matrix:

$$
\begin{array}{r}
\mathcal{N}=\left(\begin{array}{cccc}
2 \mathcal{R}^{1} \mathcal{R}^{2} \mathcal{R}^{3} & -\mathcal{R}^{2} \mathcal{R}^{3} & -\mathcal{R}^{1} \mathcal{R}^{3} & -\mathcal{R}^{1} \mathcal{R}^{2} \\
\cdots & 0 & \mathcal{R}^{3} & \mathcal{R}^{2} \\
\ldots & \ldots & 0 & \mathcal{R}^{1} \\
\cdots & \cdots & \cdots & 0
\end{array}\right) \\
+i\left(\begin{array}{cccc}
q \mathcal{I}^{1} \mathcal{I}^{2} \mathcal{I}^{3} & -\mathcal{I}^{2} \mathcal{I}^{3} \frac{\mathcal{R}^{1}}{\mathcal{I}^{1}} & -\mathcal{I}^{1} \mathcal{I}^{3} \frac{\mathcal{R}^{2}}{\mathcal{I}^{2}} & -\mathcal{I}^{1} \mathcal{I}^{2} \frac{\mathcal{R}^{3}}{\mathcal{I}^{3}} \\
\cdots & \frac{\mathcal{I}^{2} \mathcal{I}^{3}}{\mathcal{I}^{1}} & 0 & 0 \\
\cdots & \cdots & \frac{\mathcal{I}^{1} \mathcal{I}^{3}}{\mathcal{I}^{2}} & 0 \\
\cdots & \ldots & \cdots & \frac{\mathcal{I}^{1} \mathcal{I}^{2}}{\mathcal{I}^{3}}
\end{array}\right),
\end{array}
$$

where

$$
q=1+\left(\frac{\mathcal{R}^{1}}{\mathcal{I}^{1}}\right)^{2}+\left(\frac{\mathcal{R}^{2}}{\mathcal{I}^{2}}\right)^{2}+\left(\frac{\mathcal{R}^{3}}{\mathcal{I}^{3}}\right)^{2} .
$$

Note that $\mathcal{I}^{i}<0$ in the physical domain of $\operatorname{Im} \mathcal{N}<0$.

\subsection{Flux quantization on orientifolds}

Flux quantization on orientifolds is quite subtle, since there exist 3-cycles "smaller" than the ones on the torus. As defined above, the fluxes of $H_{3}$ and $F_{3}$ must belong to $H^{3}(X, \mathbb{Z})$, and respectively, the homological Poincaré duals $\left[H_{3}\right]$ and $\left[F_{3}\right]$ must belong to $H_{3}(X, \mathbb{Z})$.

Fluxes for $\epsilon=+1$ orbifold

Following the arguments used in [20], one notices that under the $\mathbb{Z}_{2} \otimes \mathbb{Z}_{2}^{\prime}$ and the $\Omega R(-1)^{F_{L}}$ action a toroidal 3 -cycle $\pi_{u}$, in a general position, is mapped to an orbit of eight toroidal 3-cycles all wrapping the same homology class on $T^{6}$. Therefore, what is usually called a bulk 3-cycle on the orientifold of $X$ is actually, from the toroidal point of view, a cycle where all $e_{\Lambda}$ and $m^{\Lambda}$ are multiples 3 of 8 . Hence the associated fluxes have $N_{\text {flux }}$ quantized in multiples of 64 . Besides these bulk cycles totally inherited from the ambient $T^{6}$, there are so-called twisted 3-cycles, which also wrap some of the 48 -cycles hidden in the various $\mathbb{Z}_{2} \times \mathbb{Z}_{2}^{\prime}$ fixed points. Note that since the twisted cycles are "shorter" than the toroidal ones, the corresponding fluxes can carry smaller quanta of $N_{\text {flux }}$. This is important for model-buliding since, as we will see later, the bulk flux contribution, which is a quantum of $N_{\text {flux }}=64$, always exceeds the negative contribution of the O3-planes to the R-R tadpole cancellation condition. Hence supersymmetric configurations can only be obtained by switching on some twisted fluxes (with $N_{\text {flux }} \leq 32$ ).

3 By that we actually mean $e_{\Lambda}^{i}$ and $m^{i \Lambda}, i=1,2$. 
Fluxes for $\epsilon=-1$ orbifold

For the model with discrete torsion the situation is a bit more subtle. In the following, we will argue that here $e_{\Lambda}$ and $m^{\Lambda}$ are only quantized in units of 4. Again arguing about the homology, $H_{3}(X, \mathbb{Z})$, we consider first 3 -cycles on the $\mathbb{Z}_{2} \otimes \mathbb{Z}_{2}^{\prime}$ orbifold space, i.e. neglecting the orientifold projection for the moment. Naively, one obtains that under the action of $\mathbb{Z}_{2} \otimes \mathbb{Z}_{2}^{\prime}$ a bulk 3-cycle on the orbifold corresponds to an orbit of four toroidal 3cycles, $4 \pi_{u}$. One can show that the intersection numbers for these bulk cycles are multiples of 4 . Since the twisted sectors do not contain any additional 3-cycles, there must exist smaller toroidal 3-cycles, as an integral basis of $H_{3}(X, \mathbb{Z})$ yields a unimodular intersection form. Thus, we conclude that there must exist 3-cycles in the orbifold, which correspond to only toroidal orbits of length two: $2 \pi_{u}$.

These shorter 3 -cycles can be seen as follows. Consider the $\theta$-twisted sector. In this sector, besides the bulk 3-cycles, we can define fractional 3-cycles, which are of the form $\pi_{u}+\pi_{t w}$, where $\pi_{u}$ denotes a toroidal cycle and $\pi_{t w}$ a 3 -cycle in the $\theta$-twisted sector. Under the action of the second $\mathbb{Z}_{2}$ such a fractional cycle is mapped to $\pi_{u}-\pi_{t w}$, so that the whole orbit under $\mathbb{Z}_{2} \otimes \mathbb{Z}_{2}^{\prime}$ gives rise to a pure toroidal cycle $2 \pi_{u}$, which is indeed what we are looking for.

Taking also the $\Omega R(-1)^{F_{L}}$ action into account, we conclude that in the $\mathbb{Z}_{2} \otimes \mathbb{Z}_{2}^{\prime}$ orientifold model with discrete torsion, the coefficients in the expansion

$$
\frac{1}{(2 \pi)^{2} \alpha^{\prime}}[G]=e_{\Lambda} A^{\Lambda}+m^{\Lambda} B_{\Lambda}
$$

are multiples of 4 . Therefore here the $N_{\text {flux }}$ is quantized in multiples of 16 , which does not exceed the contribution from the O3-planes.

\subsection{Supersymmetry breaking}

In order to discuss supersymmetry breaking, we will examine the transformations of fermions. The relevant terms depending on the 3-form background are [51,52]

$$
\begin{aligned}
& \delta \lambda \propto \mathcal{G} \epsilon+\ldots \\
& \delta \psi^{m} \propto \Gamma^{m} \mathcal{G} \bar{\epsilon}+2 \mathcal{G} \Gamma^{m} \bar{\epsilon}+\ldots
\end{aligned}
$$

where $\mathcal{G} \equiv G_{a b c} \Gamma^{a b c}$, and $\epsilon$ is the supersymmetry transformation parameter. 1 it is convenient to use the following form of $D=10,32 \times 32$ gamma matrices:

$$
\Gamma^{\mu}=I \otimes \gamma^{\mu} \quad \Gamma^{i}=\gamma^{i} \otimes \gamma_{5}
$$

4 We are using the notation of [51]. 
where $\gamma^{\mu}$ and $\gamma_{5}$ are $D=4$ gamma matrices. $I$ is the $8 \times 8$ identity matrix; $\gamma^{i}$ are $D=6$ matrices for which we adopt the representation of [53]:

$$
\begin{aligned}
\gamma^{1} & =\frac{1}{2} \sigma^{1}\left(1+\sigma^{3}\right) \otimes \mathbf{1} \otimes \mathbf{1} & \gamma^{\overline{1}} & =\frac{1}{2} \sigma^{1}\left(1-\sigma^{3}\right) \otimes \mathbf{1} \otimes \mathbf{1} \\
\gamma^{2} & =-\sigma^{3} \otimes \frac{i}{2} \sigma^{2}\left(1+\sigma^{3}\right) \otimes \mathbf{1} & \gamma^{\overline{2}} & =-\sigma^{3} \otimes \frac{i}{2} \sigma^{2}\left(1-\sigma^{3}\right) \otimes \mathbf{1} \\
\gamma^{3} & =\sigma^{3} \otimes \sigma^{3} \otimes \frac{1}{2} \sigma^{1}\left(1+\sigma^{3}\right) & \gamma^{\overline{3}} & =\sigma^{3} \otimes \sigma^{3} \otimes \frac{1}{2} \sigma^{1}\left(1-\sigma^{3}\right) .
\end{aligned}
$$

As in [53], we will denote the $\operatorname{SO}(2)$ spinor $\left(\begin{array}{l}1 \\ 0\end{array}\right)$ by + and $\left(\begin{array}{l}0 \\ 1\end{array}\right)$ by - . The $D=10$ spinor $\epsilon$ is chiral, and can be written as $\epsilon=\sum_{k=1}^{k=4}\left(\Psi^{k} \otimes \psi^{k}+\bar{\Theta}^{k} \otimes \bar{\theta}^{k}\right)$, where $\Psi$ and $\bar{\Theta}$ are righthanded and left-handed $\mathrm{SO}(6)$ spinors, respectively, i.e. containing even or odd numbers of $(-)$, while $\psi$ and $\bar{\theta}$ are similarly right- and left-handed in $D=4$. However, the orbifold projection leaves us with $\mathrm{SO}(6)$ spinors containing either only $(+)$ or only $(-)$, so in the absence of fluxes, the compactified theory is $N=2$ supersymmetric, with

$$
\epsilon=(+++) \otimes \psi+(---) \otimes \bar{\theta}
$$

which can also be written as a sum of two Majorana-Weyl spinors $\epsilon=\epsilon_{L}+i \epsilon_{R}$ with

$$
\epsilon_{A}=(+++) \otimes \eta_{A}+(---) \otimes \bar{\eta}_{A}
$$

where $A=L, R$, while $\eta_{A}$ are right-handed components of $D=4$ Majorana spinors.5

The $\Omega R(-1)^{F_{L}}$ orientifold projection leads to one more restriction on the supersymmetry parameters (4.13). In particular it relates the left- and right-moving supersymmetries because of

$$
i \epsilon_{R}=i \Gamma^{4} \ldots \Gamma^{9} \epsilon_{L}
$$

Thus, we see that the surviving spinor is

$$
\epsilon=2(---) \otimes \bar{\eta}_{L}
$$

\subsection{An example with supersymmetry}

In the first example, we consider the following flux configuration:

$$
\vec{m}^{0}=(0,4), \quad \vec{e}_{0}=(-4,0), \quad \vec{m}^{3}=(-4,0), \quad \vec{e}_{3}=(0,-4)
$$

5 Here, the indices $A=L, R$ refer to the left- and right-moving parts of the superstring and should not be confused with spacetime chirality. 
or equivalently,

$$
\frac{1}{(2 \pi)^{2} \alpha^{\prime}} G=4\left(\alpha_{0}-\tau \beta^{0}-\tau \alpha_{3}-\beta^{3}\right) .
$$

Since $m \times e=32$, we are looking for a solution of Eq. (3.15), with the period matrix given in (4.6). Indeed, one can show that the unique solution to these equations is

$$
\mathcal{T}^{1} \mathcal{T}^{2}=-1, \quad \tau=-\mathcal{T}^{3} .
$$

The superpotential is

$$
W=4 \sqrt{\mu_{3}}\left(-\tau-\mathcal{T}^{3}-\mathcal{T}^{1} \mathcal{T}^{2} \mathcal{T}^{3}-\tau \mathcal{T}^{1} \mathcal{T}^{2}\right),
$$

which vanishes for (4.18). Moreover, all derivatives satisfy

$$
D_{i} W=D_{\tau} W=W=0,
$$

so that the flux (4.17) is a pure $(2, \overline{1})$ form. This can explicitly be seen by writing the G-flux as

$$
\frac{1}{(2 \pi)^{2} \alpha^{\prime}} G=\frac{4 \mathcal{T}^{1}}{\mathcal{T}^{1}-\overline{\mathcal{T}}^{1}} a_{1}+\frac{4 \mathcal{T}^{2}}{\mathcal{T}^{2}-\overline{\mathcal{T}}^{2}} a_{2}
$$

Hence we expect that $N=1$ supersymmetry remains unbroken.

In order to verify this, we examine the supersymmetry transformations (4.9) with 6 $\mathcal{G} \propto \gamma^{\overline{1}} \gamma^{2} \gamma^{3}+\gamma^{1} \gamma^{\overline{2}} \gamma^{3}$. These variations are restricted by

$$
\gamma^{i}(---)=\gamma^{\bar{\imath}}(+++)=0 .
$$

In fact, it is easy to see that all variations under the (- - ) transformations are zero, so that the corresponding supersymmetry remains unbroken. Under the $(+++)$ transformations, the internal components $\psi^{1}$ and $\psi^{2}$ of the gravitino have non-vanishing variations; therefore the corresponding supersymmetry is broken. Note that the unbroken supersymmetry is also preserved by the orientifold projection. One can also check explicitly, that $G$ is indeed primitive.

Neglecting the orientifold projection, the fluxes under consideration provide a nice example of partial $N=2 \rightarrow N=1$ supersymmetry breaking and moduli stabilization. This partial breaking has the same origin as in the APT mechanism [54] in globally supersymmetric theories, where "non-locality" is due to the simultaneous presence of electric and magnetic Fayet-Iliopoulos terms, which can be described by a superpotential of the form (3.18). Here, we have found a flux configuration that preserves supersymmetry and fixes two combinations of the four complex moduli $\left\{\tau, \mathcal{T}^{1}, \mathcal{T}^{2}, \mathcal{T}^{3}\right\}$. In the next section, we will come back to this supersymmetry-preserving example.

\footnotetext{
6 Note that, in our conventions, $\bar{\epsilon}=(+++) \otimes \bar{\psi}+(---) \otimes \theta$.
} 


\subsection{An example with supersymmetry breaking}

As another example, we consider

$$
\vec{m}^{0}=(0,4), \quad \vec{e}_{0}=(-4,0) \quad \Rightarrow \quad \frac{1}{(2 \pi)^{2} \alpha^{\prime}} G=4\left(\alpha_{0}-\tau \beta^{0}\right) .
$$

Now (3.15) is solved by $\mathcal{R}^{1}=\mathcal{R}^{2}=\mathcal{R}^{3}=\operatorname{Re} \tau=0$ and

$$
\operatorname{Im} \tau=-\mathcal{I}^{1} \mathcal{I}^{2} \mathcal{I}^{3} .
$$

At this minimum, $D_{i} W=D_{\tau} W=0$; however, $W=-8 \tau \neq 0$. Furthermore,

$$
\frac{1}{(2 \pi)^{2} \alpha^{\prime}} G=\frac{2}{3}\left(a_{1}+a_{2}+a_{3}\right)+2 \bar{\Omega}_{3},
$$

and the flux therefore contains also a $(0, \overline{3})$ contribution. As in the previous example, the $(+++)$ supersymmetry is broken, but now in addition, the $(---)$ supersymmetry is also broken. By looking at the variations (4.9), it is easy to check that it is indeed the $(0, \overline{3})$ part of the flux that is responsible for this breaking. From the point of view of the effective supergravity theory, $N=1$ supersymmetry is broken by a vacuum expectation value of the superpotential, i.e. of the auxiliary component of the gravitational supermultiplet. This is a no-scale supersymmetry breaking, at a scale undetermined at the classical level. A detailed supergravity description of moduli stabilization and partial supersymmetry breaking in similar orientifold models has been worked out in [55].

\section{D-branes with magnetic fluxes}

So far, we have discussed the consequences of turning on the background G-fluxes. We have seen that they contribute to the R-R tadpole cancellation conditions and that a nontrivial superpotential is generated, which freezes some of the complex structure moduli. In the papers on such flux compactifications the R-R-tadpole conditions are trivially satisfied by introducing D3- and D7-branes located on top of the orientifold planes. In our case, this would lead to a consistent model, even though the massless spectrum on the branes would be non-chiral. If we have really phenomenological applications of such models in mind, we have to introduce branes in such a way that chiral fermions are generated.

A possible way of achieving this is by introducing D9-branes with abelian magnetic fluxes 45,2,201. Such configurations are T-dual (mirror-symmetric) to intersecting D6branes, which have been discussed extensively in the recent literature [1]26]. Moreover, it is known that such abelian magnetic fluxes, via the integrated Dirac-Born-Infeld (DBI) action, give rise to a D-term potential for the Kähler structure moduli $\mathcal{K}_{s}^{I}$. Therefore, one expects that turning on both G-flux and magnetic fluxes freezes both some of the complex structure moduli $\mathcal{T}^{i}$ via F-terms and Kähler structure moduli $\mathcal{K}_{s}^{I}$ via D-terms. 


\section{1. $R$ - $R$ tadpoles}

In order to cancel the 4-form and 8-form tadpoles arising from the O3- and O7-planes in the $\mathbb{Z}_{2} \times \mathbb{Z}_{2}^{\prime}$ orientifold model, we introduce D9-branes with abelian magnetic fluxes. First we notice that under the orientifold projection a D9-brane with a constant magnetic flux, $F$, is mapped into a $\overline{\mathrm{D} 9}$ brane with the opposite flux, $-F$. Therefore, one might naively expect that supersymmetry is broken by such branes. For pure D9-branes this is indeed the case. However, by turning on constant magnetic fluxes on the D9-branes, supersymmetric configurations are possible.

The Chern-Simons term for the D9- $\overline{\mathrm{D} 9}$ brane system

$$
S_{C S}=\mu_{9} \int_{\mathrm{D} 9} \operatorname{Tr}\left(e^{2 \pi \alpha^{\prime} F}\right) \wedge \sum C_{q}-\mu_{9} \int_{\overline{\mathrm{D} 9}} \operatorname{Tr}\left(e^{-2 \pi \alpha^{\prime} F}\right) \wedge \sum C_{q}
$$

introduces possible tadpoles not only for the R-R 10-form but also for lower-rank R-R forms. Here we have taken into account that at the orbifold point (away from the singular fixed points) the manifold is flat, so that the curvature contributions to the Chern-Simons term vanish. Note, that the R-R 10-form and the R-R 6-form tadpole cancels automatically in (5.1). To be more explicit, we choose $K$ stacks of $N_{a}$ D9-branes, $a=1, \ldots K$, with the abelian magnetic fluxes

$$
\mathcal{F}_{a}=2 \pi \alpha^{\prime} F_{a}=2 \pi \alpha^{\prime} \sum_{I=1}^{3} F_{a}^{I} d x^{I} \wedge d y^{I}
$$

turned on on each brane. We use the normalization $\int_{T_{I}^{2}} d x^{I} \wedge d y^{I}=1$, for each $I=1,2,3$. Considering the T-dual situation with D6-branes at angles, it is clear that we have two co-prime integers $\left(n^{I}, m^{I}\right)$ for each $T_{I}^{2}$ to specify such a configuration

$$
F_{a}^{I}=2 \pi \frac{n_{a}^{I}}{m_{a}^{I}}
$$

The integer $m^{I}$ can be interpreted as the wrapping number of the D9-brane around the 2-cycle $T_{I}^{2}$ and the second integer $n^{I}$ is the first quantized Chern class of the $U(1)$ gauge bundle

$$
c_{1}\left(F_{a}^{I}\right)=\frac{1}{2 \pi} \int_{m_{a}^{I} \times T_{I}^{2}} F_{a}^{I} d x^{I} \wedge d y^{I}=n_{a}^{I} .
$$

Here we have taken into account that the D9-brane wraps the two-dimensional torus $m^{I}$ times. Under the action of $\Omega R(-1)^{F_{L}}$ such a brane with flux is mapped to a $\overline{\mathrm{D} 9}$ brane 
with the opposite magnetic flux. Moreover, we get lower-dimensional branes by choosing for the wrapping numbers $m^{I}=0$.

So far we have only discussed the toroidal case. In the presence of discrete torsion, the orbifold space contains also 48 additional 2-cycles from the three $\mathbb{Z}_{2}$ twisted sectors, which in principle can also support non-vanishing magnetic fluxes. At the orbifold point, such a brane would correspond to a fractional D9-brane, which is also charged under twisted sector R-R fields. To keep the presentation as simple as possible, we will not consider such flux configurations in this paper and will assume that the Kähler moduli related to these twisted 2-cycles are frozen at the orbifold point, i.e. the volumes of the exceptional 2-cycles vanish. Here we only consider magnetic 2-form fluxes through the three 2-cycles inherited from the toroidal ambient space. Arguments similar to the ones given for the allowed 3-cycles on the orbifold space lead to the conclusion that such a bulk brane in the orbifold space can be described by four copies of the toroidal branes. This explains some extra factors of 4 in the formulas presented below.

From the Chern-Simons action, it is now straightforward to derive the contribution of the D9- $\overline{\mathrm{D} 9}$ branes with fluxes to the R-R tadpole cancellation condition. Taking also the contribution from the orientifold planes and the G-flux into account, we arrive at the following four conditions

$$
\begin{aligned}
8 \sum_{a} N_{a} \prod_{I} n_{a}^{I}+N_{\text {flux }} & =32 \\
8 \sum_{a} N_{a} n_{a}^{1} m_{a}^{2} m_{a}^{3} & =-32 \epsilon \\
8 \sum_{a} N_{a} m_{a}^{1} n_{a}^{2} m_{a}^{3} & =-32 \\
8 \sum_{a} N_{a} m_{a}^{1} m_{a}^{2} n_{a}^{3} & =-32
\end{aligned}
$$

Here, the perturbative orientifold with discrete torsion $(\epsilon=-1)$ is assumed to contain one set of $\mathrm{O} 7$ planes of the type $\mathrm{O} 7^{(+,+)}$.

The G-flux of course only contributes to the first line in (5.5), which is the R-R tadpole cancellation condition of the 4-form. Moreover, the 10-form and 6-form tadpoles vanish automatically due to the presence of the $\overline{\mathrm{D} 9}$ branes with opposite magnetic flux. This is consistent with the fact that these forms are projected out by $\Omega R(-1)^{F_{L}}$. 
Using the Atiyah-Singer index theorem, or employing simply the results of [2], the chiral massless spectrum transforming in the $U\left(N_{1}\right) \times \ldots \times U\left(N_{K}\right)$ gauge group is given in Table 1.

\begin{tabular}{|c|c|}
\hline Representation & Multiplicity \\
\hline$\left[\mathbf{A}_{\mathbf{a}}\right]_{L}$ & $\frac{1}{2}\left(I_{a^{\prime} a}+I_{O a}\right)$ \\
\hline$\left[\mathbf{S}_{\mathbf{a}}\right]_{L}$ & $\frac{1}{2}\left(I_{a^{\prime} a}-I_{O a}\right)$ \\
\hline$\left[\left(\mathbf{N}_{\mathbf{a}}, \overline{\mathbf{N}}_{\mathbf{b}}\right)\right]_{L}$ & $4 \prod_{I}\left(m_{a}^{I} n_{b}^{I}-n_{a}^{I} m_{b}^{I}\right)$ \\
\hline$\left[\left(\mathbf{N}_{\mathbf{a}}, \mathbf{N}_{\mathbf{b}}\right)\right]_{L}$ & $4 \prod_{I}\left(m_{a}^{I} n_{b}^{I}+n_{a}^{I} m_{b}^{I}\right)$ \\
\hline
\end{tabular}

Table 1: Chiral spectrum in $D=4$

In Table 1 we used

$$
\begin{aligned}
& I_{a^{\prime} a}=32 \prod_{I} n_{a}^{I} m_{a}^{I} \\
& I_{O a}=8 \prod_{I} m_{a}^{I}-8 \epsilon m_{a}^{1} n_{a}^{2} n_{a}^{3}-8 n_{a}^{1} m_{a}^{2} n_{a}^{3}-8 n_{a}^{1} n_{a}^{2} m_{a}^{3} .
\end{aligned}
$$

As we mentioned, extra factors of 4 appear because we only consider bulk branes.

A comment on gauge anomalies is in order at this point. For vanishing G-flux, the spectrum in Table 1 is free of four-dimensional non-abelian gauge anomalies, if the R$\mathrm{R}$ tadpole cancellation conditions are satisfied. However, for non-trivial G-fluxes this is no longer true and the chiral massless spectrum in Table 1 gives rise to the non-abelian $S U\left(N_{a}\right)^{3}$ gauge anomaly:

$$
\delta_{\Lambda} \log Z_{a}=-\frac{1}{3 !(2 \pi)^{2}} N_{\text {flux }} \prod_{I} m_{a}^{I} \int_{M^{4}}\left[F_{a} \wedge F_{a} \wedge F_{a}\right]^{(1)},
$$

where we have used the Wess-Zumino descent relation notation, i.e. for a closed gauge invariant form $Y$, we define $Y=d Y^{(0)}$ and $\delta Y^{(0)}=d Y^{(1)}$, where $\delta$ denotes a gauge variation. As pointed out in [56], this anomaly is canceled by an in-flow mechanism resulting from the following term in the Chern-Simons action of the D9-brane:

$$
S_{C S}=\mu_{9} \int_{D 9_{a}} C_{2} \wedge B \wedge \frac{1}{3 !}\left(2 \pi \alpha^{\prime}\right)^{3} F_{a} \wedge F_{a} \wedge F_{a}
$$

By the usual descent relations, and taking the wrapping number of the D9-brane into account, this leads to the anomalous gauge variation

$$
\delta_{\Lambda} S_{C S}=\frac{1}{3 !(2 \pi)^{2}} N_{f l u x} \prod_{I} m_{a}^{I} \int_{M^{4}}\left[F_{a} \wedge F_{a} \wedge F_{a}\right]^{(1)}
$$

canceling precisely the naive anomaly (5.7). 


\subsection{Supersymmetry}

In order to identify the supersymmetry preserved by D9-branes with magnetic fluxes, it is convenient to use the formalism of [57]. Applying the results of [57] to one D9-brane with magnetic fluxes, one finds that $N=1$ supersymmetry is preserved provided that

$$
\begin{aligned}
\sin \left(\varphi_{a}\right)\left(\frac{1}{2} J\right. & \left.\wedge J \wedge \mathcal{F}_{a}-\frac{1}{3 !} \mathcal{F}_{a} \wedge \mathcal{F}_{a} \wedge \mathcal{F}_{a}\right)+ \\
\cos \left(\varphi_{a}\right)\left(\frac{1}{2} J\right. & \left.\wedge \mathcal{F}_{a} \wedge \mathcal{F}_{a}-\frac{1}{3 !} J \wedge J \wedge J\right)=0
\end{aligned}
$$

for any parameter $\varphi_{a}$. Here $J$ denotes the Kähler form on the Calabi-Yau manifold. This can compactly be written as

$$
\operatorname{Im}\left(e^{-i \varphi_{a}} \Phi_{a}\right)=0
$$

with

$$
\Phi_{a}=\frac{1}{3 !}\left(\mathcal{F}_{a}+i J\right) \wedge\left(\mathcal{F}_{a}+i J\right) \wedge\left(\mathcal{F}_{a}+i J\right)
$$

In this form the supersymmetry condition looks very similar to the T-dual (mirror symmetric) condition for a 3-cycle $\Gamma$ to be volume minimizing, $\left.\operatorname{Im}\left(e^{-i \varphi} \Omega_{3}\right)\right|_{\Gamma}=0$. The second T-dual supersymmetric 3-cycle condition, namely that the 3 -cycle is lagrangian, $\left.J\right|_{\Gamma}=0$, is automatically satisfied by flat 3-cycles that are mapped to our D9-branes with constant magnetic fluxes.

Expanding the Kähler form in the string frame as

$$
J=\sum_{I} \mathcal{K}_{s}^{I} d x^{I} \wedge d y^{I}
$$

and defining the angle variables $\psi_{a}^{I}$ as

$$
\tan \psi_{a}^{I}=2 \pi \alpha^{\prime} \frac{F_{a}^{I}}{\mathcal{K}_{s}^{I}}
$$

Eq. (5.10) boils down to the familiar supersymmetry condition

$$
\sum_{I} \psi_{a}^{I}=\frac{3 \pi}{2}-\varphi_{a} \quad \bmod 2 \pi .
$$

As is well known, changing one of the angles $\psi_{a}^{I}$ and consequently also $\varphi_{a}$ by $\pi$ turns the D9-brane into a $\overline{\mathrm{D} 9}$ brane. 
Then, the supersymmetry preserved by a brane satisfying (5.15) is given by

$$
\epsilon_{R}=\Gamma_{0} \ldots \Gamma_{9} \rho(M) \epsilon_{L},
$$

where $\rho(M)$ denotes the rotation matrix by the angles $\psi_{a}^{I}$ in the spinor representation [58,59]. Thus, a D9- brane preserves the same supersymmetry as the orientifold plane (4.14), if

$$
\epsilon_{L}=i \gamma_{5} \rho(M) \epsilon_{L} .
$$

Inserting the form of the left-moving spinor (4.13), we arrive at the eigenvalue equation

$$
\rho(M) \eta_{L}=-i \eta_{L}
$$

which has a solution if

$$
\sum_{I} \psi_{a}^{I}=3 \pi / 2 \bmod 2 \pi .
$$

Therefore, the $N=1$ supersymmetry preserved by the orientifold planes corresponds to $\varphi_{o}=0$ and therefore, in order to preserve the same supersymmetry, we have to choose $\varphi_{a}=0$ for all $K$ stacks of D9-D9 branes.

\subsection{The scalar potential}

The DBI action for a stack of D9-branes with magnetic flux, when integrated over the internal volume, gives rise to a scalar potential of the form

$$
\mathcal{V}_{a}=\mu_{9} e^{-\phi} N_{a} \int_{X} d^{6} x \sqrt{\operatorname{det}\left[G+2 \pi \alpha^{\prime} F\right]},
$$

which for constant abelian fluxes is exact to all orders in $\alpha^{\prime}$. This potential holds for both supersymmetric and non-supersymmetric D9-branes but for supersymmetric gauge fluxes satisfying (5.10) the computation simplifies, as the square-root in (5.20) simplifies. In analogy to the T-dual situation for calibrated 3-cycles, for a supersymmetric D9-brane, this action can be expressed as

$$
\mathcal{V}_{a}=\mu_{9} e^{-\phi} N_{a} \int_{X} \operatorname{Re}\left(e^{-i \varphi_{a}} \Phi_{a}\right) .
$$

Since we have computed the scalar potential in the closed string sector in the Einstein frame we should also shift to the Einstein frame in the open string sector. Defining the 
Einstein frame Kähler moduli as $\mathcal{K}_{E}=e^{-\phi / 2} \mathcal{K}_{s}$, we can bring the scalar potential for $K$ stacks of supersymmetric D9- $\overline{\mathrm{D} 9}$ branes with angles $\varphi_{a}$ into the suggestive form

$$
\begin{aligned}
\mathcal{V}_{D 9}= & 8 \sum_{a} \mathcal{V}_{a} \\
= & 8 \mu_{3} \sum_{a} \cos \varphi_{a} N_{a} \prod_{I} n_{a}^{I}-8 \mu_{7} e^{\phi} \sum_{a} \cos \varphi_{a} N_{a} \sum_{I \neq J \neq K} n_{a}^{I} m_{a}^{J} m_{a}^{K} \mathcal{K}_{E}^{J} \mathcal{K}_{E}^{K} \\
& -8 \mu_{9} e^{\frac{3}{2} \phi} \sum_{a} \sin \varphi_{a} N_{a} \prod_{I} m_{a}^{I} \mathcal{K}_{E}^{I}+8 \mu_{5} e^{\frac{1}{2} \phi} \sum_{a} \sin \varphi_{a} N_{a} \sum_{I \neq J \neq K} n_{a}^{I} n_{a}^{J} m_{a}^{K} \mathcal{K}_{E}^{K} .
\end{aligned}
$$

Thus we see that for generic angles $\varphi_{a}$, one gets effective D9-, D7-, D5- and D3-brane tensions. Note that the extra factors of $\left(2 \pi \alpha^{\prime}\right)$ in the magnetic flux $\mathcal{F}$ and the powers of the dilaton arrange themselves in just the right way to give the effective Einstein frame Dp-brane tensions.

Moreover, (5.22) is correct for both D9- and $\overline{\mathrm{D} 9}$-branes by defining $\varphi_{a}$ appropriately. Starting for instance with a supersymmetric D9-branes with quantum numbers $\left(n_{a}^{I}, m_{a}^{I}\right)$ and angle $\varphi_{a}$, reflecting an odd number of such pairs gives the anti-brane for which we must choose $-\varphi_{a}$ in $(5.22)$.

Adding also the contributions from the O3- and O7-planes

$$
\mathcal{V}_{O}^{E}=-32 \mu_{3}-32 \mu_{7} e^{\phi}\left(\epsilon \mathcal{K}_{E}^{2} \mathcal{K}_{E}^{3}+\mathcal{K}_{E}^{1} \mathcal{K}_{E}^{2}+\mathcal{K}_{E}^{1} \mathcal{K}_{E}^{3}\right)
$$

and the topological term from the flux-induced potential

$$
\mathcal{V}_{\text {flux }}=\mu_{3} N_{\text {flux }}
$$

and, in addition, invoking the R-R tadpole cancellation conditions (5.5), we can write the combined potential $\mathcal{V}_{D 9}+\mathcal{V}_{O}^{E}+\mathcal{V}_{\text {flux }}$ as

$$
\begin{aligned}
\mathcal{V}_{F I}= & 8 \mu_{3} \sum_{a}\left(\cos \varphi_{a}-1\right) N_{a} \prod_{I} n_{a}^{I}-8 \mu_{7} e^{\phi} \sum_{a}\left(\cos \varphi_{a}-1\right) N_{a} \sum_{I \neq J \neq K} n_{a}^{I} m_{a}^{J} m_{a}^{K} \mathcal{K}_{E}^{J} \mathcal{K}_{E}^{K} \\
& -8 \mu_{9} e^{\frac{3}{2} \phi} \sum_{a} \sin \varphi_{a} N_{a} \prod_{I} m_{a}^{I} \mathcal{K}_{E}^{I}+8 \mu_{5} e^{\frac{1}{2} \phi} \sum_{a} \sin \varphi_{a} N_{a} \sum_{I \neq J \neq K} n_{a}^{I} n_{a}^{J} m_{a}^{K} \mathcal{K}_{E}^{K} .
\end{aligned}
$$

7 Here we assume that $N_{\text {flux }}$ is positive; all equations can be consistently rewritten for negative $N_{\text {flux }}$. 
As was discussed in [16], in the effective four-dimensional theory, such a potential originates from the Fayet-Iliopolous terms associated to the $K$ abelian $U(1) \subset U\left(N_{a}\right)$ subgroups. By writing the potential as

$$
\mathcal{V}_{F I}=\sum_{a} \frac{N_{a}}{2 g_{a}^{2}} \xi_{a}^{2}
$$

one can identify the Fayet-Iliopolous terms $\xi_{a}$. This computation shows that the topological term from the flux-induced potential is crucial indeed and participates in the cancellation of Fayet-Iliopoulos terms in the D-term potential.

The positive-definite D-term potential vanishes precisely when the magnetic flux preserves supersymmetry, which means that $\operatorname{Im}\left(\Phi_{a}\right)=0$ for all $K$ stacks of D9-branes. This leads to the following constraint for the string frame Kähler moduli:

$$
-\prod_{I} \mathcal{K}_{s}^{I} m_{a}^{I}+\left(4 \pi^{2} \alpha^{\prime}\right)^{2} \sum_{I \neq J \neq K} \mathcal{K}_{s}^{I} m_{a}^{I} n_{a}^{J} n_{a}^{K}=0
$$

for all stacks of D9-branes. In general, one has more conditions than Kähler moduli, so that this condition drastically constrains the model目.

One might wonder whether the gauge fluxes also induce a superpotential of the form

$$
W=\int \Omega_{3} \wedge \omega_{Y M}
$$

where $\omega_{Y M}$ denotes the Chern-Simons form

$$
\omega_{Y M}=\sum_{a} A_{a} \wedge F_{a} .
$$

Here, it should be recalled that (5.28) arises from the general superpotential $W=\int \Omega_{3} \wedge G$ after taking into account that, in general, gauge fluxes on D9-branes induce a source for G-fluxes via the equation

$$
d G \sim \sum_{a} F_{a} \wedge F_{a}
$$

In fact, if the right-hand side happened to be non-zero, this would invalidate our whole reasoning, as $G$ would have to be combined with $\omega_{Y M}$ to give a closed 3-form (as in Type I or in the heterotic string). However, in our orientifold example, the contributions to the right-hand side of (5.30) from the D9-branes and their image $\overline{\mathrm{D} 9}$ branes just cancel, so that the magnetic fluxes only induce a D-term potential.

8 Note that actually in the D-term potential VEVs for charged fields can in principle cancel the Fayet-Iliopolous term and lead to new minima of the scalar potential [46]. However, this means that D-branes recombine and that the gauge and matter sector of the theory changes. For this not to happen we set all charged open string moduli in the D-term to zero by hand. Then supersymmetry gives rise to the condition (5.27) 


\subsection{Examples}

\subsubsection{A supersymmetric brane configuration}

Here we discuss a supersymmetric brane configuration. Choosing the orbifold without discrete torsion, $\epsilon=+1$, and switching on a G-flux 9 with $N_{\text {flux }}=32$ we introduce two stacks of four D9-branes with the quantum numbers

$$
\begin{aligned}
1^{\text {st }} \text { stack }:\left(n^{I}, m^{I}\right) & =\{(0,1),(1,-1),(1,-1)\} \\
2^{n d} \text { stack }:\left(n^{I}, m^{I}\right) & =\{(1,0),(0,1),(0,-1)\} .
\end{aligned}
$$

One realizes that all four R-R tadpole cancellation conditions are satisfied. The chiral spectrum on the D9- $\overline{\mathrm{D} 9}$ branes consists of four chiral multiplets in the $(\overline{4}, 4)$ representation and another four chiral multiplets in the $(\overline{\mathbf{4}}, \overline{\mathbf{4}})$ representation of the $U(4) \times U(4)$ gauge group. Note that the second stack of branes has $m^{(1)}=0$ on the first torus and therefore can be considered as D7-branes localized on the first $T^{2}$. Moreover, as expected from the general anomaly formula (5.7), only the first $S U\left(N_{a}\right)$ gauge factor is anomalous.

The second stack of branes is supersymmetric for any choice of the Kähler moduli, while the first stack yields the constraint

$$
\mathcal{K}_{s}^{(2)} \mathcal{K}_{s}^{(3)}=\left(4 \pi^{2} \alpha^{\prime}\right)^{2}
$$

Thus, the number of unfrozen Kähler parameters from the untwisted sector is reduced to two. We expect that one combination of the 4 -form superpartners, $C^{I}$, also gets a mass via axionic couplings from the Chern-Simons action [6, [6]. Note, that in these examples the overall volume of $T^{6}$ is not frozen.

\subsubsection{A non-supersymmetric brane configuration}

Consider the supersymmetric G-flux discussed in Section 4.4. Since $N_{\text {flux }}=32$, the four-form tadpole cancellation condition is already saturated without any D3-brane charges. We are dealing now with the model with discrete torsion, $\epsilon=-1$, which contains the $\mathrm{O}^{(+,+)}$planes. We introduce the following two stacks of four D9-branes with the quantum numbers

$$
\begin{aligned}
1^{s t} \text { stack }:\left(n^{I}, m^{I}\right) & =\{(0,1),(1,-1),(1,-1)\} \\
2^{n d} \text { stack }:\left(n^{I}, m^{I}\right) & =\{(1,0),(0,-1),(0,-1)\} .
\end{aligned}
$$

9 As explained before, such a configuration requires twisted 3-cycles. We assume that the complex stucture moduli can be stabilized in a supersymmetric vacuum, like in the example discussed in Section 4.4 . 
Indeed the R-R tadpole cancellation conditions are satisfied, where the second stack can be seen as $\overline{\mathrm{D} 7}$-branes, which have been introduced to cancel the $\mathrm{R}-\mathrm{R}$ charge of the $\mathrm{O} 7^{(+,+)}$ plane.

The gauge group is $U(4) \times U(4)$ and the chiral spectrum on the D9- $\overline{\mathrm{D} 9}$ branes consists of four chiral fermions in the $(\mathbf{4}, \overline{\mathbf{4}})$ representation, four chiral fermions in the $(\mathbf{4}, \mathbf{4})$ representation and eight chiral fermions in the $(\mathbf{6}, \mathbf{1})+(\overline{\mathbf{1 0}}, \mathbf{1})$ representation.

We can make the FI-term of the first $U(1) \subset U(4)$ vanish by satisfying the constraint

$$
\mathcal{K}_{s}^{(2)} \mathcal{K}_{s}^{(3)}=\left(4 \pi^{2} \alpha^{\prime}\right)^{2}
$$

However, in contrast to the previous example, the scalar potential (FI-term) for the second gauge factor does not vanish, but takes the value

$$
\mathcal{V}_{F I, 2}=64 \mu_{7} \mathcal{K}_{s}^{(2)} \mathcal{K}_{s}^{(3)}=64 \mu_{3}
$$

It is beyond our scope to discuss this potential in more detail; it is clear that non-trivial magnetic fluxes can fix part of the Kähler moduli.

These simple examples show that a combination of G-flux and magnetic fluxes on D9$\overline{\mathrm{D} 9}$ branes allows some of the conceptual constraints for phenomenologically interesting string models to be satisfied. On the one hand, similar to what takes place in intersecting brane worlds, one can obtain a chiral spectrum on the branes. On the other hand, the Gflux can fix some or maybe even all complex structure moduli via an F-term potential, and the abelian magnetic fluxes freeze some or even all of the Kähler moduli via an effective Dterm potential. Furthermore, the remaining $N=1$ supersymmetry can be spontaneously broken in the large radius limit in a no-scale manner, provided that one chooses a suitable G-flux configuration, like in the example described in Section 4.5.

Of course, for bulk branes we obtain, in addition, open string moduli related to continuous Wilson lines on the D9-branes along the internal directions. These are not frozen in the leading-order approximation we are discussing here. One way to freeze them already at leading order would be to also turn on magnetic fluxes through the $\mathbb{Z}_{2} \times \mathbb{Z}_{2}^{\prime}$ fixed points. These would give rise to fractional D9-branes with frozen open string moduli. It might be that the back-reaction on the geometry freezes (some of) these moduli too. 


\section{Conclusions}

In this work, we have pointed out that by combining ideas from Calabi-Yau compactifications with background fluxes and from intersecting brane-world models, it is possible to freeze both complex structure moduli and Kähler moduli. The resulting gauge/supergravity theory has many phenomenologically appealing properties: chiral spectrum, partial supersymmetry breaking and, eventually, complete supersymmetry breaking in a no-scale manner, with a vanishing cosmological constant. We have illustrated these issues on a simple example provided by the $\mathbb{Z}_{2} \times \mathbb{Z}_{2}^{\prime}$ toroidal orbifold. Of course, these concepts are more general and can in principle be applied to any Calabi-Yau orientifold model with O3-planes.

A very important point is that our computations are valid only in the leading order of string perturbation theory. Since the superpotential depends only on the complex structure moduli, it is exact to all orders in $\alpha^{\prime}$ (although $\alpha^{\prime}$ corrections to the Kähler potential will eventually also change the F-term scalar potential). In addition, our computation for the D9-branes was exact to all orders in $\alpha^{\prime}$, as we used the complete DBI action.

Taking the back-reaction of the G-flux into account, it is known that it leads to a warping of the Calabi-Yau geometry, while leaving the dilaton constant. This seems to be promising; however, the D9-branes with flux and the O7-planes do not cancel their R-R charges and tensions locally (which was essential for generating chiral asymmetry) so that their back-reaction will dramatically change the background geometry and will make the dilaton vary over the internal space. Thus, at this level, we cannot trust any longer the computation done in this work. As we know from F-theory, the background is presumably no longer Calabi-Yau; nevertheless, the hope is that the string background adjusts itself in such a way that the number of stabilized moduli remains determined by the leading order terms, and that the gauge group and the chiral matter content are not changed. It would be interesting to understand in more detail what the back-reaction is and what other moduli might be frozen by it.

Eventually, it may be worthwhile to establish duality relations to other types of flux compactifications, in particular to Type IIA orientifolds, to M-theory or to the heterotic superstring. 


\section{Acknowledgements}

We are grateful to Carlo Angelantonj, Ignatios Antoniadis, Sergio Ferrara, Gianfranco Pradisi, Stephan Stieberger, Angel M. Uranga, Pierre Vanhove and Anders Westerberg for very useful discussions. T.R.T. is grateful to the Institute of Physics at Humboldt University in Berlin, where this work was initiated, for its kind hospitality. His research is supported in part by the National Science Foundation under grant PHY-99-01057. The work of R.B. and D.L. is supported in part by the EC under the RTN project HPRN-CT2000-00131. 


\section{References}

[1] M. Berkooz, M. R. Douglas and R. G. Leigh, Branes Intersecting at Angles, Nucl. Phys. B 480 (1996) 265, hep-th/9606139.

[2] R. Blumenhagen, L. Görlich, B. Körs and D. Lüst, Noncommutative Compactifications of Type I Strings on Tori with Magnetic Background Flux, JHEP 0010 (2000) 006, hep-th/0007024.

[3] C. Angelantonj, I. Antoniadis, E. Dudas and A. Sagnotti, Type I Strings on Magnetized Orbifolds and Brane Transmutation, Phys. Lett. B 489 (2000) 223, hep-th/0007090.

[4] R. Blumenhagen, L. Görlich, B. Körs and D. Lüst, Magnetic Flux in Toroidal Type I Compactification, Fortsch. Phys. 49 (2001) 591, hep-th/0010198.

[5] C. Angelantonj and A. Sagnotti, Type I Vacua and Brane Transmutation, hepth/0010279.

[6] G. Aldazabal, S. Franco, L. E. Ibañez, R. Rabadan and A. M. Uranga, D = 4 Chiral String Compactifications from Intersecting Branes, J. Math. Phys. 42 (2001) 3103, hep-th/0011073.

[7] G. Aldazabal, S. Franco, L. E. Ibañez, R. Rabadan and A. M. Uranga, Intersecting Brane Worlds, JHEP 0102 (2001) 047, hep-ph/0011132.

[8] R. Blumenhagen, B. Körs and D. Lüst, Type I Strings with $F$ and B-Flux, JHEP 0102 (2001) 030, hep-th/0012156.

[9] L. E. Ibañez, F. Marchesano and R. Rabadan, Getting just the Standard Model at Intersecting Branes, JHEP 0111 (2001) 002, hep-th/0105155.

[10] S. Förste, G. Honecker and R. Schreyer, Orientifolds with Branes at Angles, JHEP 0106 (2001) 004, hep-th/0105208.

[11] R. Rabadan, Branes at Angles, Torons, Stability and Supersymmetry, Nucl. Phys. B 620 (2002) 152, hep-th/0107036.

[12] R. Blumenhagen, B. Körs, D. Lüst and T. Ott, The Standard Model from Stable Intersecting Brane World Orbifolds, Nucl. Phys. B 616 (2001) 3, hep-th/0107138.

[13] M. Cvetic, G. Shiu and A. M. Uranga, Three-Family Supersymmetric Standard-like Models from Intersecting Brane Worlds, Phys. Rev. Lett. 87 (2001) 201801, hepth/0107143.

[14] M. Cvetic, G. Shiu and A. M. Uranga, Chiral Four-Dimensional N=1 Supersymmetric Type IIA Orientifolds from Intersecting D6-Branes, Nucl. Phys. B 615 (2001) 3, hepth/0107166.

[15] D. Bailin, G. V. Kraniotis and A. Love, Standard-like Models from Intersecting D4branes, Phys. Lett. B 530 (2002) 202, hep-th/0108131.

[16] D. Cremades, L. E. Ibañez and F. Marchesano, SUSY Quivers, Intersecting Branes and the Modest Hierarchy Problem, JHEP 0207 (2002) 009, hep-th/0201205. 
[17] R. Blumenhagen, B. Körs, D. Lüst and T. Ott, Hybrid Inflation in Intersecting Brane Worlds, Nucl. Phys. B641 (2002) 235, hep-th/0202124.

[18] D. Cremades, L. E. Ibañez and F. Marchesano, Intersecting Brane Models of Particle Physics and the Higgs Mechanism, JHEP 0207 (2002) 022, hep-th/0203160.

[19] C. Kokorelis, GUT Model Hierarchies from Intersecting Branes, JHEP 0208 (2002) 018, hep-th/0203187.

[20] R. Blumenhagen, V. Braun, B. Körs and D. Lüst, Orientifolds of K3 and Calabi-Yau Manifolds with Intersecting D-branes, JHEP 0207 (2002) 026, hep-th/0206038.

[21] A.M. Uranga, Local models for intersecting brane worlds, hep-th/0208014.

[22] R. Blumenhagen, V. Braun, B. Körs and D. Lüst, The Standard Model on the Quintic, hep-th/0210083.

[23] G. Pradisi, Magnetized (Shift-) Orientifolds, hep-th/0210088.

[24] R. Blumenhagen, L. Görlich and T. Ott, Supersymmetric Intersecting Branes on the Type IIA $T^{6} / Z_{4}$ orientifold, JHEP 0301 (2003) 021, hep-th/0211059.

[25] D. Cremades, L. E. Ibanez and F. Marchesano, Yukawa couplings in intersecting Dbrane models, hep-th/0302105.

[26] D. Lüst and S. Stieberger, Gauge Threshold Corrections in Intersecting Brane World Models, hep-th/0302221.

[27] J. Polchinski and A. Strominger, New Vacua for Type II String Theory, Phys. Lett. B 388 (1996) 736, hep-th/9510227.

[28] T.R. Taylor and C. Vafa, RR flux on Calabi-Yau and partial supersymmetry breaking, Phys. Lett. B 474, 130 (2000), hep-th/9912152.

[29] K. Dasgupta, G. Rajesh and S. Sethi, M theory, orientifolds and G-flux, JHEP 9908, 023 (1999), hep-th/9908088.

[30] P. Mayr, On Supersymmetry Breaking in String Theory and its Realization in Brane Worlds, Nucl.Phys. B593 (2001) 99, hep-th/0003198.

[31] G. Curio, A. Klemm, D. Lüst and S. Theisen, On the Vacuum Structure of Type II String Compactifications on Calabi-Yau Spaces with H-Fluxes, Nucl. Phys. B609 (2001) 3, hep-th/0012213.

[32] S. B. Giddings, S. Kachru and J. Polchinski, Hierarchies from Fluxes in String Compactifications, hep-th/0105097.

[33] G. Curio, A. Klemm, B. Körs and D. Lüst, Fluxes in Heterotic and Type II String Compactifications, Nucl. Phys. B620 (2002) 237, hep-th/0106155.

[34] G. Curio, B. Körs and D. Lüst, Fluxes and Branes in Type II Vacua and M-theory Geometry with G(2) and Spin(7) Holonomy, Nucl. Phys. B636 (2002) 197, hepth/0111165.

[35] S. Kachru, M. Schulz and S. Trivedi, Moduli Stabilization from Fluxes in a Simple IIB Orientifold, hep-th/0201028. 
[36] A.R. Frey and J. Polchinski, N=3 Warped Compactifications, Phys. Rev. D65 (2002) 126009, hep-th/0201029.

[37] K. Becker, M. Becker, M. Haack and J. Louis, Supersymmetry Breaking and alpha'Corrections to Flux Induced Potentials, JHEP 0206 (2002) 060, hep-th/0204254.

[38] R. D'Auria, S. Ferrara and S. Vaula, $N=4$ gauged supergravity and a IIB orientifold with fluxes, New J.Phys. 4 (2002) 71, hep-th/0206241.

[39] O. DeWolfe and S.B. Giddings, Scales and hierarchies in warped compactifications and brane worlds, hep-th/0208123.

[40] A.R. Frey and A. Mazumdar, 3-Form Induced Potentials, Dilaton Stabilization, and Running Moduli, hep-th/0210254.

[41] S. Gurrieri, J. Louis, A. Micu and D. Waldram, Mirror Symmetry in Generalized Calabi-Yau Compactifications, hep-th/0211102.

[42] S. Kachru, M. Schulz, P.K. Tripathy and S. Trivedi, New Supersymmetric String Compactifications, hep-th/0211182.

[43] P.K. Tripathy and S.P. Trivedi, Compactification with Flux on K3 and Tori, hepth/0301139.

[44] S. Kachru, R. Kallosh, A. Linde and S.P. Trivedi, de Sitter Vacua in String Theory, hep-th/0301240.

[45] C. Bachas, A Way to Break Supersymmetry, hep-th/9503030.

[46] I. Antoniadis, E. Gava, K.S. Narain and T.R. Taylor, Duality in superstring compactifications with magnetic field backgrounds, Nucl. Phys. B 511 (1998) 611, hepth/9708075.

[47] C. Angelantonj, I. Antoniadis, G. D'Appollonio, E. Dudas and A. Sagnotti, Type I vacua with brane supersymmetry breaking, Nucl. Phys. B572 (2000) 36, hepth/9911081.

[48] A. Ceresole, R. D'Auria and S. Ferrara, The Symplectic Structure of N=2 Supergravity and its Central Extension, Nucl. Phys. Proc. Suppl. 46 (1996) 67, hep-th/9509160.

[49] S. Gukov, C. Vafa and E. Witten, CFT's From Calabi-Yau Four-folds, Nucl. Phys. B584 (2000) 69, Erratum-ibid. B608 (2001) 477, hep-th/9906070.

[50] S. Gukov, Solitons, superpotentials and calibrations, Nucl. Phys. B574 (2000) 169, hep-th/9911011.

[51] P.S. Howe and P.C. West, The Complete N=2, D=10 Supergravity, Nucl. Phys. B 238 (1984) 181.

[52] M. Grana and J. Polchinski, Gauge/Gravity Duals with Holomorphic Dilaton, Phys. Rev. D65 (2002) 126005, hep-th/0106014.

[53] J.J. Atick and A. Sen, Covariant one Loop Fermion Emission Amplitudes in Closed String Theories, Nucl. Phys. B293 (1987) 317.

[54] I. Antoniadis, H. Partouche and T.R. Taylor, Spontaneous Breaking of N=2 Global Supersymmetry, Phys. Lett. B 372 (1996) 83, hep-th/9512006. 
[55] S. Ferrara and M. Porrati, $N=1$ no-scale supergravity from IIB orientifolds, Phys. Lett. B 545 (2002) 411, hep-th/0207135; L. Andrianopoli, R. D'Auria, S. Ferrara and M. A. Lledo, Gauged extended supergravity without cosmological constant: No-scale structure and supersymmetry breaking, hep-th/0212141; L. Andrianopoli, R. D'Auria, S. Ferrara and M. A. Lledo, $N=2$ super-Higgs, $N=1$ Poincaré vacua and quaternionic geometry, JHEP 0301 (2003) 045, hep-th/0212236; L. Andrianopoli, R. D'Auria, S. Ferrara and M. A. Lledo, 4-D supergravity analysis of Type IIB vacua on $K_{3} \times T^{2} / Z_{2}$, hep-th/0302174.

[56] A. M. Uranga, D-brane, Fluxes and Chirality, hep-th/0201221.

[57] M. Mariño, R. Minasian, G. W. Moore and A. Strominger, Nonlinear Instantons from Supersymmetric p-branes, JHEP 0001 (2000) 005, hep-th/9911206.

[58] M. Mihailescu, I.Y. Park and T.A. Tran, D-branes as Solitons of an $N=1, D=10$ Non-commutative Gauge Theory, Phys.Rev. D64 (2001) 046006, hep-th/0011079.

[59] E. Witten, BPS Bound States of D0-D6 and D0-D8 Systems in a B-Field, JHEP 0204 (2002) 012, hep-th/0012054. 